\title{
COMPOSIÇÃO CORPORAL E OCORRÊNCIA DE LESÃO POR PRESSÃO: REVISÃO INTEGRATIVA
}

\author{
BODY COMPOSITION AND PRESSURE INJURY \\ OCCURRENCE: AN INTEGRATIVE REVIEW
}

\section{COMPOSICIÓN CORPORAL Y OCURRENCIA DE LESIÓN POR PRESIÓN: REVISIÓN INTEGRADORA}

\author{
Jéssica Gomes da Silva ${ }^{1}$ \\ Karoline Faria de Oliveira ${ }^{2}$ \\ Maria Beatriz Guimarães Ferreira ${ }^{3}$ \\ Flávia Ana Pacheco ${ }^{4}$ \\ Isadora Braga Calegari ${ }^{4}$ \\ Maria Helena Barbosa ${ }^{5}$
}

Como citar este artigo: Silva JG, Oliveira KF, Ferreira MBG, Pacheco FA, Calegari IB, Barbosa MH. Composição corporal e ocorrência de lesão por pressão: revisão integrativa. Rev baiana enferm. 2019;33:e28790.

Objetivo: analisar a relação entre a composição corporal com a distribuição de pressão de interface sob proeminências ósseas e a ocorrência de lesão por pressão. Método: revisão integrativa da literatura realizada nas bases de dados PubMed, CINAHL, Lilacs, Embase ${ }^{\circledR}$, Scopus e Web of Science, publicados entre 1990 e julho de 2018. Resultados: foram selecionados nove estudos e classificados em duas categorias temáticas. A primeira categoria abordou a relação da lesão por pressão com classificação nutricional e a segunda a relação da composição corporal com a pressão de interface. O risco de lesão por pressão esteve relacionado à diminuição da massa magra, da quantidade de água corporal e ao índice de massa corporal extremo; além de maior incidência em idosos institucionalizados com redução de massa gorda. Conclusão: houve relação da composição corporal com a ocorrência de lesão por pressão, apresentando divergências entre adultos e idosos.

Descritores: Composição Corporal. Impedância Elétrica. Lesão por Pressão. Posicionamento do Paciente. Segurança do Paciente.

Objective: to analyze the relationship between body composition and interface pressure distribution under bony prominences and the occurrence of pressure injury. Method: this is an integrative literature review conducted in the PubMed, CINAHL, Lilacs, Embase ${ }^{\circledR}$, Scopus, and Web of Science databases, published between 1990 and July 2018. Results: nine studies were selected and classified into two thematic categories. The first category addressed the relationship of pressure injury with nutritional classification and the second the relationship of body composition with interface pressure. The risk of pressure injury was related to the decrease in lean body mass, body water content and extreme body mass index; besides higher incidence in institutionalized elderly with fat mass reduction. Conclusion: there was a relationship between body composition and the occurrence of pressure injury, showing differences between adults and the elderly.

Descriptors: Body Composition. Electrical Impedance. Pressure Injury. Patient Positioning. Patient Safety.

\footnotetext{
Enfermeira. Pesquisadora Independente. Uberaba, Minas Gerais, Brasil.

Enfermeira. Doutora em Atenção à Saúde. Enfermeira do Instituto de Ciências da Saúde da Universidade Federal do Triângulo Mineiro. Uberaba, Minas Gerais, Brasil. Enfermeira. Doutora em Ciências. Professora Adjunta da Universidade Federal de Uberlândia. Uberaba, Minas Gerais, Brasil.

Enfermeira. Universidade Federal do Triângulo Mineiro. Uberaba, Minas Gerais, Brasil.

Enfermeira. Doutora em Enfermagem na Saúde do Adulto. Professora Associada. Universidade Federal do Triângulo Mineiro. Uberaba, Minas Gerais, Brasil. mhelena33।@hotmail.com
} 
Objetivo: analizar la relación entre la composición corporal con la distribución de presión de interface debajo de prominencias óseas y la ocurrencia de lesión por presión. Método: revisión integradora de la literatura realizada en las bases de datos PubMed, CINAHL, Lilacs, Embase ${ }^{\circledR}$, Scopus e Web of Science, publicados entre 1990 y julio de 2018. Resultados: se seleccionaron nueve estudios y se clasificaron en dos categorías temáticas. La primera categoría abordó la relación de la lesión por presión con clasificación nutricional y la segunda, la relación de la composición corporal con la presión de interface. El riesgo de lesión por presión estuvo relacionado a la disminución de la masa magra, de la cantidad de agua corporal y al índice de masa corporal extremo; además de mayor incidencia en ancianos institucionalizados con reducción de masa grasa. Conclusión: hubo relación de la composición corporal con la ocurrencia de lesión por presión, presentando divergencias entre adultos y ancianos.

Descriptores: Composición Corporal. Impedancia Eléctrica. Lesión por Presión. Posicionamiento del Paciente. Seguridad del Paciente.

\section{Introdução}

A despeito da ampla disponibilização de recursos tecnológicos para a prevenção de lesão por pressão (LPP), como o uso de superfícies de suporte $^{(1)}$, a ocorrência desse tipo de lesão ainda é considerada um problema de alta incidência relacionado à assistência à saúde ${ }^{(2)}$. Essa incidência está diretamente associada à qualidade do atendimento, à segurança do paciente, ao tempo de internação e aos custos hospitalares ${ }^{(3-4)}$.

Dentre os fatores de risco para o desenvolvimento da LPP, os aspectos nutricionais são descritos como fatores causais indiretos ${ }^{(5)}$. Classificações nutricionais extremas, como magreza ou obesidade, são consideradas fatores de risco para a ocorrência de LPP. A primeira, pelo aumento da exposição das proeminências ósseas do paciente, por reduzir as áreas de contato expostas à pressão de interface. A segunda, por potencializar complicações decorrentes do posicionamento $^{(6-7)}$.

O índice de massa corporal (IMC) é o indicador utilizado para a realização da classificação nutricional de magreza e obesidade. Entretanto, outros indicadores da composição corporal, como a razão entre massa magra, massa gorda e água corporal, poderiam ser utilizados para determinar a influência na ocorrência de lesões. Esses podem modificar a exposição da proeminência óssea de acordo com o tipo de tecido a que essa extremidade está exposta.

Frente ao exposto, o objetivo do presente estudo foi analisar a relação entre a composição corporal com a distribuição de pressão de interface sob proeminências ósseas e a ocorrência de lesão por pressão. Verificar se essa relação proporcionará subsídios para que o enfermeiro identifique pacientes com maior risco de LPP e elabore um plano de cuidados fundamentado na incorporação de resultados de pesquisas à prática clínica.

\section{Método}

Revisão integrativa da literatura, realizada por meio das seguintes etapas: estabelecimento da questão de pesquisa, amostragem ou busca na literatura de estudos primários, extração de dados, avaliação dos estudos e inclusão na revisão, análise e interpretação dos resultados e síntese do conhecimento ${ }^{(8)}$. As questões norteadoras da revisão integrativa foram: Quais as evidências disponíveis em relação à associação da ocorrência de lesão por pressão com a composição corporal? Existe relação da composição corporal com a redistribuição da pressão de interface exercida sobre as proeminências ósseas?

Para levantamento dos estudos, foram utilizadas as bases de dados US National Library of Medicine (Pubmed), Cumulative Index to Nursing and Allied Health Literature (Cinahl), Literatura Latino Americana e do Caribe em Ciências da Saúde (Lilacs), Excerpta Medica dataBASE (Embase $\left.{ }^{\circledR}\right)$, Scopus Elsevier e Web of Science. Para a definição das bases de dados considerou-se o quantitativo de indexação de artigos da área da 
saúde, bases que contemplam estudos primários e temáticas relacionadas à enfermagem.

A seleção dos descritores ocorreu de acordo com as ferramentas de busca das respectivas bases de dados primários, sendo utilizados termos do Medical Subject Headings (Mesh), para busca nas bases PubMed, Embase ${ }^{\circledR}$, Web of Science e Scopus; termos de Descritores em Ciências da Saúde (DeCS), para a base de dados Lilacs; e termos do Cinabl Headings, para a base de dados Cinahl. Os descritores utilizados - body composition, pressure ulcer, patient positioning, decubitus - foram analisados isoladamente e com auxílio do operador booleano "AND" entre eles. Vale ressaltar que nem todos foram utilizados em todas as bases por divergência de nomenclatura entre elas. Os descritores foram agrupados em dois ou três termos, para que a estratégia de busca fosse sensibilizada e não subestimasse os resultados esperados. A estratégia de busca que permitiu a identificação de estudos primários incluídos na revisão é apresentada no Quadro 1.

Quadro 1 - Estratégia de busca concretizada que permitiu a identificação dos estudos primários na revisão integrativa

\begin{tabular}{|l|c|c|c|}
\hline \multicolumn{4}{|c|}{ Estratégia de Busca } \\
\hline Base de dados & $\begin{array}{c}\text { Pressure Ulcer } \\
\text { /Decubitus AND } \\
\text { Body Composition }\end{array}$ & $\begin{array}{c}\text { Pressure Ulcer } \\
\text { / Decubitus AND } \\
\text { Patient Positioning }\end{array}$ & $\begin{array}{c}\text { Pressure Ulcer / Decubitus } \\
\text { AND Body Composition AND } \\
\text { Patient Positioning }\end{array}$ \\
\hline PubMed & 6 & 0 & 0 \\
\hline Cinahl & 2 & 0 & 0 \\
\hline Lilacs & 0 & 0 & 0 \\
\hline Scopus & 1 & 0 & 0 \\
\hline Web of Science & 0 & 0 & 0 \\
\hline EmBase & 0 & 0 & 0 \\
\hline
\end{tabular}

Fonte: Elaboração própria.

Para inclusão dos artigos, consideraram-se os seguintes critérios: estudos que retratavam a temática, respondendo às questões norteadoras; publicados entre 1990 e julho de 2018; e apresentados na íntegra em versão português, inglês ou espanhol. Os trabalhos encontrados foram classificados em relação à hierarquia de evidências segundo o Sistema de Classificação para a Hierarquia das Evidências de Intervenção / Questões de Tratamento ${ }^{(9)}$. Foram excluídos os artigos que não abordaram a relação da composição corporal ou da pressão de interface com a ocorrência de LPP, além de editorial, carta-resposta, estudos de revisão narrativa de literatura/revisão tradicional, métodos de revisão e que apresentassem inconsistências na metodologia proposta.
Um total de 183 documentos foram identificados nas seis bases de dados investigadas. A seleção dos artigos foi norteada segundo as diretrizes Principais Itens para Relatar Revisões sistemáticas e Meta-análises (PRISMA), conforme apresentado na Figura 1. A primeira seleção dos artigos foi realizada por meio da leitura de título e resumo. Conforme os critérios de seleção da revisão integrativa, foram selecionados nove estudos. A ordem das bases de dados analisadas foi PubMed, Lilacs, Embase ${ }^{\circledR}$, Cinahl, Scopus e Web of Science. A ordem de exclusões seguiu os seguintes critérios: artigo repetido, fora dos anos estabelecidos, fora dos idiomas estabelecidos, fora do tema e artigos de revisão da literatura. 
Figura 1 - Fluxo da seleção dos artigos da revisão, segundo diretrizes do PRISMA

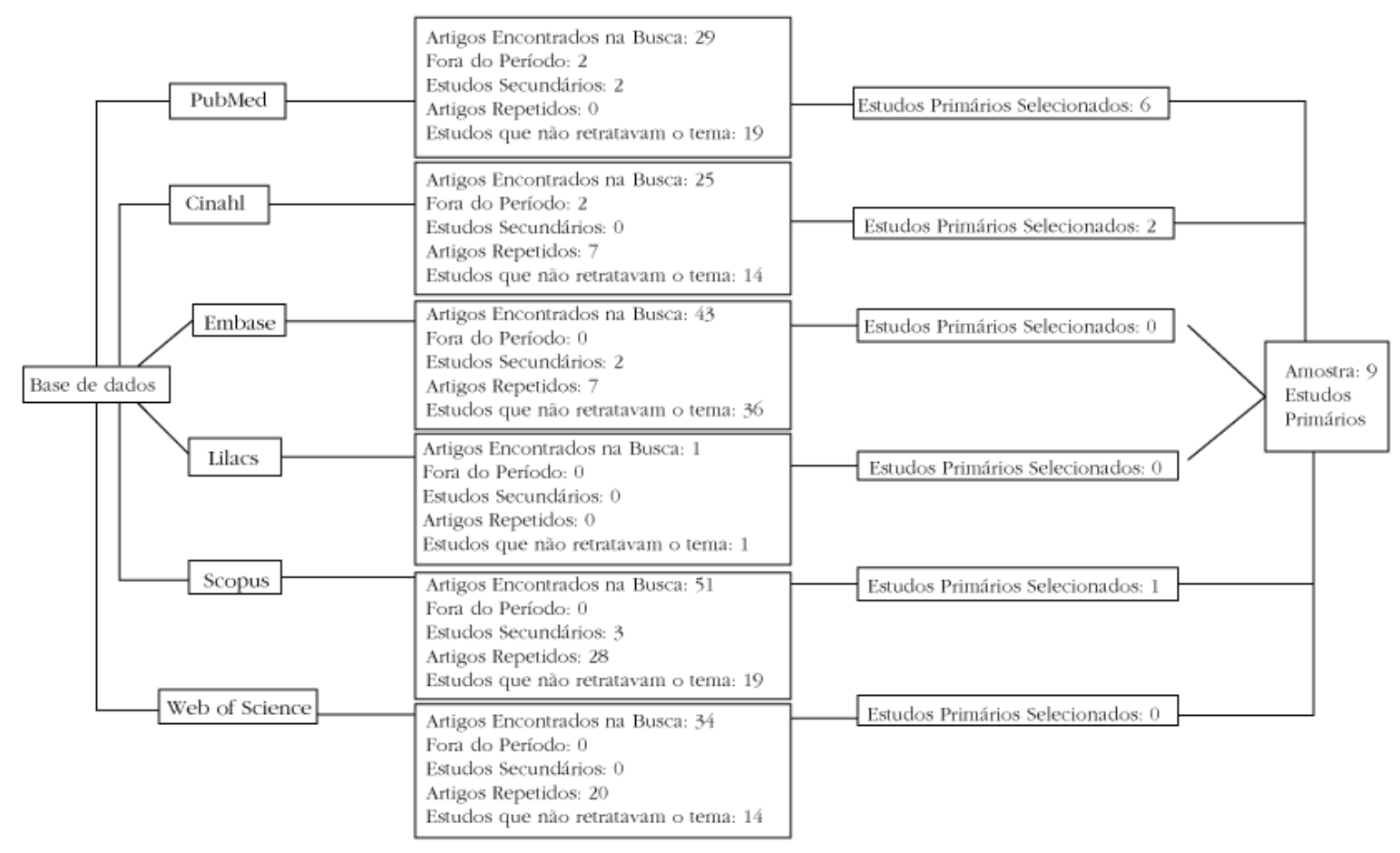

Fonte: Elaboração própria.

A extração dos dados dos estudos primários selecionados foi executada utilizando-se um instrumento adaptado de coleta de dados, proposto e validado(10). Tal instrumento contempla a identificação do artigo, ano e local do estudo, características metodológicas, avaliação do rigor metodológico, nível de evidência, principais resultados e discussões relacionadas à questão investigada.

Três pesquisadores independentemente sintetizaram os resultados neste estudo. Quando não houve consistência dos resultados sintetizados, uma discussão foi realizada entre os investigadores até se chegar a um consenso. As informações extraídas foram tabuladas para a síntese dos dados.

A avaliação dos tipos de estudos selecionados foi feita com base nos conceitos de estudiosos de metodologia científica ${ }^{(11)}$, os quais os classificam em duas abordagens metodológicas: quantitativa e qualitativa. Os estudos encontrados foram classificados quanto à hierarquia das evidências ${ }^{(9)}$. $\mathrm{O}$ nível de evidência não foi considerado um critério de exclusão, devido à existência de poucos estudos com melhores evidências e a heterogeneidade dos delineamentos.

A análise dos resultados foi feita de forma descritiva, de modo que foi apresentada uma síntese de cada um dos estudos primários incluídos na presente revisão.

\section{Resultados}

Dos nove artigos incluídos na revisão, oito foram publicados no idioma inglês e um no espanhol. Em relação aos periódicos, dois eram relacionados a aspectos nutricionais, dois a princípios mecânicos e de reabilitação, outros três com enfoque sobre a gerontologia e apenas um específico de feridas crônicas e agudas. Apenas um estudo era proveniente de periódico brasileiro: Revista Brasileira de Geriatria e Gerontologia.

Os estudos foram classificados quanto à hierarquia de evidências científicas: 4 (44,44\%) foram classificados como nível de evidência 3; 2 (22,22\%) como nível de evidência 4; e 3 (33,33\%) 
como nível de evidência 6. Todos os estudos foram classificados como quantitativos.

A população avaliada nos estudos totalizou 805 participantes, dentre os quais alguns pacientes com e sem lesão, adultos, idosos e voluntários saudáveis.

Os estudos primários incluídos foram classificados em duas categorias temáticas, que abordavam a relação da LPP com a classificação nutricional (categoria 1) e com a pressão de interface (categoria 2). Os estudos 1 ao 5 e o estudo 7 foram agrupados na primeira categoria; os estudos 6, 8 e 9 na segunda categoria. A seguir, uma síntese de cada um dos artigos incluídos na revisão é apresentada detalhadamente.

O estudo 1 foi realizado na Itália e teve como objetivo investigar o estado nutricional, a composição corporal e o metabolismo energético em 52 idosas institucionalizadas distribuídas em dois grupos, a saber: 23 idosas com LPP em estágio avançado e 29 sem lesão. Também identificaram possíveis condições hipermetabólicas e sua relação com a área e o volume da lesão. Os pacientes foram avaliados quanto ao índice de massa corporal (IMC), massa livre de gordura, massa gorda, despesa de energia de repouso e área e volume da LPP. Estas foram avaliadas, respectivamente, em centímetros e pela injeção de soro fisiológico, o qual foi mensurado pela sua quantidade. Os resultados evidenciaram que IMC e quantidades de massa livre de gordura e gorda foram semelhantes em ambos os grupos, entretanto as pacientes com LPP apresentaram maior gasto energético. Quando comparados os grupos, as pacientes com lesão não tiveram diminuição da massa livre de gordura, mas apresentaram carência proteica ${ }^{(12)}$.

Com o objetivo de determinar as causas e os possíveis fatores de risco que predispõem à LPP, o estudo 2 foi realizado em Portugal com 40 idosos institucionalizados, dos quais 12 apresentavam LPP e 28 não. Para tal, avaliou-se composição corporal, dados laboratoriais, estado nutricional e comorbidades. Os resultados demonstraram que, na análise da composição corporal, o grupo sem lesão apresentou valores inferiores de gordura corporal e maiores de albumina sérica. Houve diferença estatisticamente significativa $(\mathrm{p}=0,05)$ entre os grupos para hidratação corporal, sendo maior nos pacientes sem $\operatorname{LPP}^{(13)}$.

O terceiro estudo foi realizado na Suíça com 32 pacientes idosos provenientes de um hospital geriátrico após dez dias de internação e classificados com baixo risco para desenvolver a lesão. Investigou-se a viscoelasticidade e a hidratação da pele em diferentes áreas propensas à LPP correlacionando esses dados com medidas realizadas no antebraço. Os pacientes foram avaliados em relação às seguintes características: propriedades viscoelásticas da pele e hidratação do antebraço interno, do sacro e do trocânter; composição corporal por meio do IMC e da impedância bioelétrica; parâmetros laboratoriais com coleta de sangue em jejum; risco para desenvolvimento de LPP pela Escala de Braden; e nutrição pelo Questionário de Mini Avaliação Nutricional. Os resultados evidenciaram que mulheres e homens não diferiram quanto à idade, IMC, pontuação nutricional, parâmetros laboratoriais e Braden. Entretanto, os homens apresentaram maior quantidade de água corporal total e massa magra, bem como menor massa gorda. Quanto às propriedades viscoelásticas, a elasticidade da pele diferiu conforme o local. A elasticidade geral, tanto nas mulheres quanto nos homens foi maior no trocânter, sacro e antebraço. As elasticidades pura ou biológica ou a viscoelasticidade da pele não diferiram para ambos os sexos. Ao correlacionar as propriedades biofísicas da pele nos diferentes sítios corporais, não houve associação com a hidratação da pele; entretanto, a elasticidade geral do antebraço correlacionou-se significativamente com as medidas de elasticidade do trocânter e do sacro. Este estudo piloto evidenciou que mudanças de propriedades mecânicas - como a redução da elasticidade e do turgor da pele - relacionadas à idade é um fator de risco para o desenvolvimento de lesões por pressão superficiais $^{(14)}$.

Os autores do estudo 4 tinham como hipótese que a gordura corporal poderia prevenir os riscos relacionados à desnutrição e morbimortalidade. Assim, objetivaram avaliar a relação entre 
a composição corporal e a morbimortalidade em idosos hospitalizados. Para tal, estabeleceram a relação entre a massa gorda e a morbimortalidade, considerando como complicações as LPP e as infecções (pneumonia, infecção do trato urinário, exceto cistite, septicemia, erisipela, enterite, artrite infecciosa e parotidite). A justificativa para tal deve-se ao fato de que essas são complicações relacionadas à desnutrição hospitalar, prevalentes em idosos hospitalizados e que contribuem para altas taxas de morbimortalidade. $\mathrm{O}$ estudo foi realizado na França e participaram 125 idosos que foram avaliados quanto ao estado nutricional (peso, IMC e composição corporal), albuminemia e proteína $\mathrm{C}$ reativa. Esses pacientes foram avaliados na admissão e acompanhados por seis meses, para identificação de possíveis complicações, por meio de uma técnica que os autores consideram padrão-ouro: absortometria de raio-x de dupla energia e análise de impedância bioelétrica (BIA). Durante os seis meses de acompanhamento, 31 (25\%) pacientes tiveram complicações infecciosas, LPP ou ambos. Onze pacientes (9\%) desenvolveram essas lesões e dois desses estavam associados a complicações infecciosas. Quatorze pacientes apresentavam-se com mobilidade totalmente prejudicada no momento da admissão. Dentre esses, 3 (21\%) evoluíram com LPP. Entretanto, o risco para desenvolver essas lesões não foi significativamente aumentado em casos de imobilidade. Além disso, os índices de massa gorda não diferiram entre os pacientes imóveis e os que não apresentavam comprometimento na mobilidade. Os pacientes foram estratificados em três categorias de acordo com o índice de massa gorda: abaixo do percentil 30, entre 30 e 70 e acima do percentil 70 . $\mathrm{O}$ risco de morte $(\mathrm{OR}=0,30 ; \mathrm{p}=0,049)$ e o risco de morte ou de complicações $(\mathrm{OR}=0,25 ; \mathrm{p}=0,02)$ foram significativamente menores para pacientes com um índice de massa gorda acima do percentil 70. O estudo evidenciou que a massa gorda foi associada a uma diminuição do risco para eventos adversos, incluindo as lesões por pressão. Há que se ressaltar que, de fato, a obesidade é um fator de risco para os adultos e que os resultados do estudo somente se aplicam a idosos hospitalizados ${ }^{(15)}$.

Com o objetivo de prevenir a desnutrição, o estudo 5, realizado na Alemanha, teve o objetivo de identificar uma ferramenta de triagem para determinar o estado nutricional. Para tal, durante 8 meses, 484 idosos com diversas doenças em condições subagudas foram selecionados após 48 horas de internação hospitalar em uma instituição geriátrica alemã. Os pacientes foram avaliados em diferentes aspectos: LPP conforme o estadiamento da European Pressure Ulcer Advisory Panel (EPUAP); risco para desenvolver LPP pela escala de Norton; estado nutricional pela Mini Avaliação Nutricional; medidas antropométricas e análise de composição corporal por impedância bioelétrica. Os resultados evidenciaram que os pacientes com LPP tiveram significativamente um tempo maior de internação do que os que não apresentaram $(\mathrm{p}<0,001)$. A prevalência de LPP foi de $16,7 \%$ e a média do risco de desenvolver LPP, conforme escala de Norton, foi de 20 pontos, classificada como risco moderado. Pela Mini Avaliação Nutricional, o estado nutricional dos pacientes portadores de LPP foi significativamente menor quando comparado aos pacientes sem as lesões. Além disso, o estado nutricional não influenciou a composição corporal dos pacientes com LPP. Massa gorda, água corporal total e massa celular diminuíram significativamente em pacientes com LPP, de modo que a massa celular foi reduzida em cerca de 20\% nos pacientes com lesões. Houve também uma correlação significativa entre o risco de desenvolver LPP e a massa celular corporal. O instrumento de Mini Avaliação Nutricional constituiu-se como uma ferramenta de rastreio e avaliação, além de ser de fácil aplicação para determinar o estado nutricional de pacientes geriátricos com LPP na admissão hospitalar ${ }^{(16)}$.

Com a intenção de avaliar a correlação entre a pressão de interface de assento e os fatores de composição corporal de peso, IMC, músculo esquelético e água corporal, o estudo 6, realizado na Coreia, investigou os efeitos do tipo de almofada de ar e várias posturas de assento sobre as 
mudanças de pressão de interface do assento. Para tal, comparou os resultados entre um grupo de 20 pacientes com lesão de medula e outros 20 pacientes sem lesões musculoesqueléticas ou nervosas. Para comparar a composição corporal entre os grupos controle e lesados, massa muscular esquelética total e segmentada, foram avaliados peso, IMC e água corporal total e segmentada. A pressão de interface de assento foi mensurada em quatro situações, a saber: sem almofada, com almofada de baixo custo e com almofadas de ar de $5 \mathrm{~cm}$ e $10 \mathrm{~cm}$. Além disso, a pressão foi mensurada em três posições: ereta, posterior inclinada em $20^{\circ}$ e em flexão para frente em $20^{\circ}$, por 10 segundos em cada posição. As massas musculares total e dos membros inferiores do grupo controle foram significativamente maior quando comparadas ao grupo de lesados, entretanto a massa muscular do tronco foi semelhante. A gordura corporal foi significativamente maior no grupo de lesados, mas a água corporal dos membros inferiores foi maior para o grupo controle. Peso corporal, IMC e água do corpo do tronco não foram estatisticamente diferentes. Todas as variáveis de composição de massa corporal não apresentaram correlações significativas com a pressão de interface em ambos os grupos. Os três tipos de almofada evidenciaram uma redução significativa na pressão de interface de assento. A mensuração das três posições diferentes, com e sem almofada de ar, não evidenciou diferença de pressão estatisticamente significativa entre as posturas. Assim, o estudo concluiu que a composição de massa corporal não apresentou efeito direto sobre a pressão de interface de assento para nenhum dos grupos. Há que se ressaltar que a redução de massa musculoesquelética e de água inerentes aos pacientes com lesão medular tem influência sobre a ocorrência de $\operatorname{LPP}^{(17)}$.

O estudo 7, realizado nos Estados Unidos, foi projetado para determinar se a impedância bioelétrica (BIA) poderia medir as características dos tecidos locais, o que identificaria pacientes com risco de desenvolver LPP. Além da BIA, foi realizada biópsia do tecido em grupos de indivíduos classificados como alto risco para desenvolver LP ( $\mathrm{n}=10)$, de acordo com a escala de Braden, e em indivíduos saudáveis $(n=10)$, os quais representaram o grupo controle. A análise de BIA total não apresentou diferença significativa entre os grupos. No entanto, a análise por segmentos apresentou reatância, resistência e ângulo de fase médios significativamente menores no grupo de alto risco em comparação ao grupo controle $(\mathrm{p}<0,01, \mathrm{p}<0,01, \mathrm{p}<0,05$, respectivamente). Não foi possível determinar diferenças dermopatológicas, pois as biópsias foram interrompidas devido ao risco associado ao procedimento. Em relação à análise de regressão linear múltipla, a reatância trocantérica local do tecido foi o indicador mais relacionado ao alto risco de desenvolver $\operatorname{LPP}^{(18)}$.

A pesquisa 8 , realizada no Reino Unido, de delineamento quase experimental, objetivou propor um modelo matemático para prever o risco de formação de LPP combinando a avaliação de risco de Waterlow e parâmetros fisiológicos (idade, peso e altura). A amostra de conveniência foi composta por 11 indivíduos adultos, com IMC de $25,04 \pm 3,01 \mathrm{~kg} / \mathrm{m}^{2}$. Foram avaliadas a pressão de interface das proeminências ósseas do calcanhar, da sacral e do cotovelo com o indivíduo em decúbito horizontal, em condições de relaxamento. A pressão de cada área foi verificada por quatro sensores Flexiforce (modelo A201) em cada região avaliada, além da idade, peso, altura e IMC. O modelo matemático foi criado, utilizando pontuações referentes a cada critério avaliado. Permite que um usuário defina a propriedade da superfície juntamente com os parâmetros fisiológicos do indivíduo. Evidenciou-se, em diferentes faixas etárias e proeminências ósseas, que a porcentagem de risco para formação de LPP é maior para IMC extremo, sendo menor para indivíduos eutróficos. Além disso, o risco foi maior em região sacral em comparação às regiões do cotovelo e calcanhar. Em relação à pressão de interface analisada sobre polímero viscoelástico, houve um aumento da pressão de interface, à medida que se elevou o IMC, exceto para faixa etária de 35 anos, que apresentou pressão de interface maior em eutróficos em relação ao sobrepeso. Ao se comparar 
as faixas etárias, para a mesma classificação nutricional, observou-se um comportamento heterogêneo da pressão de interface, no qual os valores de pressão de interface foram divergentes para um mesmo $\mathrm{IMC}^{(19)}$.

Analisar a sensibilidade à rigidez dos tecidos moles das nádegas, utilizando o modelo de elementos finitos na posição sentada, constituiu o objetivo do estudo 9, realizado na França. O modelo foi construído com base na segmentação de uma tomografia computadorizada, que forneceu as superfícies da pele, dos músculos e dos ossos. Estas avaliações mostraram que a camada de pele teve pouca influência sobre as estirpes máximas. Isto se deve provavelmente ao fato de que tal camada é muito fina e bastante rígida. Por outro lado, devido às grandes espessuras e rigidez inferior, as camadas de gordura e músculo apresentaram muito mais influência. A análise de sensibilidade evidenciou que as medidas de deformação Von Mises (VM) máximas estão localizadas principalmente abaixo da tuberosidade isquiática, na camada de gordura próxima à interface músculo/gordura. Esse tecido será, consequentemente, predisposto a mais LPP. As medidas de deformação VM máximas aparecem ocasionalmente dentro da camada muscular perto da interface osso/músculo, mas apenas quando o módulo de Young dos músculos é semelhante ao módulo de Young da gordura, o que é provavelmente o caso para paraplégicos ou pessoas idosas ${ }^{(20)}$.

Os Quadros 2 e 3, a seguir, apresentam a caracterização dos artigos em objetivos, delineamento de pesquisa, número de sujeitos envolvidos, descrição dos principais resultados e conclusões.

Quadro 2 - Síntese dos artigos selecionados na busca, por título (ano), objetivos e delineamento/número de pacientes

(continua)

\begin{tabular}{|c|c|c|}
\hline Título (Ano) & Objetivos & $\begin{array}{c}\text { Delineamento/ número } \\
\text { de pacientes }\end{array}$ \\
\hline $\begin{array}{l}\text { 1. Resting energy expenditure and } \\
\text { body composition in bedridden } \\
\text { institutionalized elderly women with } \\
\text { advanced-stage pressure sores } \\
\text { (2007) }\end{array}$ & $\begin{array}{l}\text { Investigar estado nutricional, } \\
\text { composição corporal e } \\
\text { metabolismo energético em idosas } \\
\text { institucionalizadas com e sem lesão. }\end{array}$ & $\begin{array}{l}\text { Estudo quantitativo, descritivo, } \\
\text { comparativo / } 52 \text { idosas ( } 23 \\
\text { com LPP em estágio avançado } \\
\text { e } 29 \text { sem lesão). }\end{array}$ \\
\hline $\begin{array}{l}\text { 2. Mini nutritional assessment and } \\
\text { screening scores are associated with } \\
\text { nutritional indicators in elderly people } \\
\text { with pressure ulcers }{ }^{(14)} \\
\text { (2008) }\end{array}$ & $\begin{array}{l}\text { Determinar causas e fatores de risco } \\
\text { que predispõem a LPP. }\end{array}$ & $\begin{array}{l}\text { Estudo descritivo / } 40 \\
\text { pacientes ( } 12 \text { com LPP e } 28 \\
\text { sem lesão). }\end{array}$ \\
\hline $\begin{array}{l}\text { 3. Assessment of biophysical skin } \\
\text { properties at different body sites in } \\
\text { hospitalized old patients: results of a } \\
\text { pilot study }{ }^{(15)} \\
(2012)\end{array}$ & $\begin{array}{l}\text { Investigar se a viscoelasticidade, } \\
\text { hidratação ou fricção diferem entre } \\
\text { áreas importantes para o risco de } \\
\text { desenvolver LP. }\end{array}$ & $\begin{array}{l}\text { Estudo piloto, descritivo, } \\
\text { comparativo / } 32 \text { idosos ( } 14 \\
\text { homens e } 18 \text { mulheres) sem } \\
\text { lesões e com baixo risco para } \\
\text { desenvolver LP. }\end{array}$ \\
\hline $\begin{array}{l}\text { 4. Fat mass protects hospitalized } \\
\text { elderly persons against morbidity and } \\
\text { mortality } \\
\text { (2009) }\end{array}$ & $\begin{array}{l}\text { Avaliar a relação entre composição } \\
\text { corporal e morbimortalidade em } \\
\text { idosos hospitalizados }\end{array}$ & $\begin{array}{l}\text { Follow-up, descritivo / } 125 \\
\text { idosos acompanhados por seis } \\
\text { meses. }\end{array}$ \\
\hline $\begin{array}{l}\text { 5. Nutrition status and pressure } \\
\text { ulcer: what we need for nutrition } \\
\text { screening } \\
(2007)\end{array}$ & $\begin{array}{l}\text { Comparar diferentes ferramentas } \\
\text { de triagem para avaliar o estado } \\
\text { nutricional. }\end{array}$ & $\begin{array}{l}\text { Descritivo / } 484 \text { idosos } \\
\text { com diversas doenças em } \\
\text { condições subagudas. }\end{array}$ \\
\hline $\begin{array}{l}\text { 6. The Effects of Body Mass } \\
\text { Composition and Cushion Type on } \\
\text { Seat-Interface Pressure in Spinal Cord } \\
\text { Injured Patients }{ }^{(18)} \\
\text { (2015) }\end{array}$ & $\begin{array}{l}\text { Investigar efeitos da composição da } \\
\text { massa corporal e do tipo almofada } \\
\text { na pressão da interface do assento } \\
\text { em pacientes com e sem lesão } \\
\text { medular. }\end{array}$ & $\begin{array}{l}\text { Quase experimental / } 40 \\
\text { pacientes ( } 20 \text { lesados } \\
\text { medulares e } 20 \text { saudáveis). }\end{array}$ \\
\hline
\end{tabular}


Quadro 2 - Síntese dos artigos selecionados na busca, por título (ano), objetivos e delineamento/número de pacientes

\begin{tabular}{|l|l|l|}
\hline \multicolumn{1}{|c|}{ Título (Ano) } & \multicolumn{1}{|c|}{ Objetivos } & \multicolumn{1}{c|}{$\begin{array}{c}\text { Delineamento/ número } \\
\text { de pacientes }\end{array}$} \\
\hline $\begin{array}{l}\text { 7. Bioelectrical impedance as a } \\
\text { discriminator of pressure ulcer risk } \\
\text { (1996) }\end{array}$ & $\begin{array}{l}\text { Identificar características objetivas } \\
\text { da estrutura e função dos tecidos } \\
\text { associados ao risco de LPP. }\end{array}$ & $\begin{array}{l}\text { Experimental Laboratorial / 20 } \\
\text { pacientes (10 com alto risco } \\
\text { para LPP e 10 voluntários } \\
\text { saudáveis). }\end{array}$ \\
\hline $\begin{array}{l}\text { 8. Modelling of pressure ulcer (PU) } \\
\text { risk prediction system } \\
\text { (2015) }\end{array}$ & $\begin{array}{l}\text { Elaborar um modelo matemático } \\
\text { para prever o risco de } \\
\text { desenvolvimento de LPP. }\end{array}$ & $\begin{array}{l}\text { Quase experimental / 11 } \\
\text { voluntários. }\end{array}$ \\
\hline $\begin{array}{l}\text { 9. Biomechanical modeling to prevent } \\
\text { ischial pressure ulcers } \\
\text { (2014) }\end{array}$ & $\begin{array}{l}\text { Analisar a sensibilidade à rigidez dos } \\
\text { tecidos moles das nádegas utilizando } \\
\text { O modelo de elementos finitos na } \\
\text { posição sentada. }\end{array}$ & $\begin{array}{l}\text { Experimental Laboratorial } \\
\text { / tomografia de um jovem } \\
\text { saudável masculino. }\end{array}$ \\
\hline
\end{tabular}

Fonte: Elaboração própria.

Quadro 3 - Síntese dos artigos selecionados na busca, por descrição e desfechos

(continua)

\begin{tabular}{|c|c|c|}
\hline Título (Ano) & Descrição & Desfechos \\
\hline $\begin{array}{l}\text { 1. Resting energy expenditure } \\
\text { and body composition in } \\
\text { bedridden institutionalized } \\
\text { elderly women with } \\
\text { advanced-stage pressure } \\
\text { sores }{ }^{(13)} \\
(2007)\end{array}$ & $\begin{array}{l}\text { Análise bioquímica, IMC, massa } \\
\text { livre de gordura, massa gorda, } \\
\text { despesa de energia de repouso } \\
\text { e área e volume da LPP foram } \\
\text { avaliados. }\end{array}$ & $\begin{array}{l}\text { IMC, massa magra e massa gorda foram } \\
\text { semelhantes entre ambos os grupos. } \\
\text { Pacientes com LPP apresentaram maior } \\
\text { gasto energético e carência proteica. }\end{array}$ \\
\hline $\begin{array}{l}\text { 2. Mini nutritional assessment } \\
\text { and screening scores are } \\
\text { associated with nutritional } \\
\text { indicators in elderly people } \\
\text { with pressure ulcers } \\
\text { (2008) }\end{array}$ & $\begin{array}{l}\text { Avaliação de composição corporal, } \\
\text { dados laboratoriais, estado } \\
\text { nutricional e comorbidades. }\end{array}$ & $\begin{array}{l}\text { Pacientes sem lesão apresentaram valores } \\
\text { inferiores de gordura corporal e maiores } \\
\text { de albumina sérica e hidratação corporal. }\end{array}$ \\
\hline $\begin{array}{l}\text { 3. Assessment of biophysical } \\
\text { skin properties at different } \\
\text { body sites in hospitalized } \\
\text { old patients: results of a pilot } \\
\text { study }{ }^{(15)} \\
(2012)\end{array}$ & $\begin{array}{l}\text { Propriedades viscoelásticas da } \\
\text { pele e hidratação, composição } \\
\text { corporal, análise laboratorial, } \\
\text { risco para desenvolvimento de } \\
\text { LPP e estado nutricional foram } \\
\text { determinados. }\end{array}$ & $\begin{array}{l}\text { Homens apresentaram maior quantidade } \\
\text { de água corporal total e massa magra, } \\
\text { bem como menor massa gorda. Redução } \\
\text { da elasticidade e do turgor da pele } \\
\text { relacionadas à idade é um fator de risco } \\
\text { para desenvolver LPP. }\end{array}$ \\
\hline $\begin{array}{l}\text { 4. Fat mass protects } \\
\text { hospitalized elderly persons } \\
\text { against morbidity and } \\
\text { mortality } \\
\text { (2009) }\end{array}$ & $\begin{array}{l}\text { Avaliou-se composição corporal, } \\
\text { albuminemia, proteína C reativa e } \\
\text { desenvolvimento de complicações } \\
\text { (LP e infecções). }\end{array}$ & $\begin{array}{l}\text { O risco de morte ou de complicações } \\
\text { foi menor para pacientes com índice de } \\
\text { massa gorda acima do percentil } 70 \text {. Massa } \\
\text { gorda foi associada à diminuição do risco } \\
\text { para LPP. }\end{array}$ \\
\hline $\begin{array}{l}\text { 5. Nutrition status and } \\
\text { pressure ulcer: what we need } \\
\text { for nutrition screening }{ }^{(17)} \\
\text { (2007) }\end{array}$ & $\begin{array}{l}\text { Avaliação do estado nutricional, } \\
\text { impedância bioelétrica, } \\
\text { atividades de vida diária, risco e } \\
\text { estadiamento da LP. }\end{array}$ & $\begin{array}{l}\text { Portadores de LPP apresentaram maior } \\
\text { tempo de internação, menor estado } \\
\text { nutricional e diminuição de massa gorda, } \\
\text { água corporal total e massa celular. Mini } \\
\text { Avaliação Nutricional pode determinar o } \\
\text { estado nutricional de idosos com LP. }\end{array}$ \\
\hline $\begin{array}{l}\text { 6. The Effects of Body Mass } \\
\text { Composition and Cushion } \\
\text { Type on Seat-Interface } \\
\text { Pressure in Spinal Cord } \\
\text { Injured Patients }{ }^{(18)} \\
\text { (2015) }\end{array}$ & $\begin{array}{l}\text { Composição corporal e pressão } \\
\text { de interface foram avaliadas. }\end{array}$ & $\begin{array}{l}\text { Composição de massa corporal não } \\
\text { apresentou efeito direto sobre a pressão } \\
\text { de interface de assento para nenhum dos } \\
\text { grupos. }\end{array}$ \\
\hline
\end{tabular}


Quadro 3 - Síntese dos artigos selecionados na busca, por descrição e desfechos

\begin{tabular}{|l|l|l|}
\hline \multicolumn{1}{|c|}{ Título (Ano) } & \multicolumn{1}{c|}{ Descrição } & \multicolumn{1}{c|}{ Desfechos } \\
\hline $\begin{array}{l}\text { 7. Bioelectrical impedance as } \\
\text { a discriminator of pressure } \\
\text { ulcer risk } \\
\text { (1996) }\end{array}$ & $\begin{array}{l}\text { Análise de impedância bioelétrica } \\
\text { e biópsia do tecido. }\end{array}$ & $\begin{array}{l}\text { Valores de impedância bioelétrica por } \\
\text { seguimentos tiveram relação com risco } \\
\text { de LPP, destaque para região trocantérica. } \\
\text { Biópsias de tecido não diferiram entre os } \\
\text { grupos. }\end{array}$ \\
\hline $\begin{array}{l}\text { 8. Modelling of pressure ulcer } \\
\text { (PU) risk prediction system } 20) \\
\text { (2015) }\end{array}$ & $\begin{array}{l}\text { Mensuração da pressão de } \\
\text { interface de proeminências ósseas } \\
\text { (calcanhar, sacral e cotovelo), } \\
\text { além de avaliações de idade, } \\
\text { peso, altura e IMC. }\end{array}$ & $\begin{array}{l}\text { O risco para LPP foi maior para IMCs } \\
\text { extremos e na região sacral. Houve um } \\
\text { aumento da pressão de interface à medida } \\
\text { que se elevava o IMC, exceto para faixa } \\
\text { etária de 35 anos. O modelo permite que } \\
\text { seja definida a propriedade da superfície } \\
\text { com os parâmetros fisiológicos do } \\
\text { indivíduo. }\end{array}$ \\
\hline $\begin{array}{l}\text { 9. Biomechanical modeling } \\
\text { to prevent ischial pressure } \\
\text { ulcers } \\
\text { (201) }\end{array}$ & $\begin{array}{l}\text { Criação da malha de elementos } \\
\text { finitos baseada na tomografia } \\
\text { de um adulto jovem do sexo } \\
\text { masculino. Avaliados os efeitos } \\
\text { da pressão de interface em três } \\
\text { posturas diferentes. }\end{array}$ & $\begin{array}{l}\text { A gordura e a camada muscular possui } \\
\text { influência importante nas variações } \\
\text { de deformação gerada pelas tensões, } \\
\text { enquanto a pele é menos influente. A } \\
\text { simulação de diferentes posturas de } \\
\text { assento com alteração da espessura da } \\
\text { camada muscular resultou em variações } \\
\text { das tensões internas. }\end{array}$ \\
\hline
\end{tabular}

Fonte: Elaboração própria.

\section{Discussão}

Os estudos primários incluídos na primeira categoria ressaltaram aspectos específicos referentes ao estado nutricional e desenvolvimento de lesões por pressão. A terapia nutricional com alto teor de proteínas e nutrientes imunomoduladores é recomendada para o tratamento de LPP. A National Pressure Ulcer Advisory Panel (NPUAP) recomenda de 1,2 a 2 g de proteínas por quilo de peso corporal do paciente ${ }^{(21)}$.

Um estudo controlado realizado com 23 pacientes portadores de LPP em estágios II, III ou IV evidenciou que o uso de suplementação proteica com aminoácidos especializados melhorou a viabilidade do tecido da lesão após duas semanas para os pacientes do grupo tratamento $(n=11)$, embora não tenha evidenciado diferenças na redução do tamanho das lesões para ambos os grupos, tratamento e controle ${ }^{(22)}$.

Em contrapartida, outro estudo realizado com 200 adultos malnutridos portadores de LPP em estágios II, III e IV evidenciou que a suplementação com a fórmula enriquecida com proteínas para grupo tratamento $(n=101)$ resultou em maior redução na área da LPP e seu uso por oito semanas promoveu melhora na cicatrização das lesões ${ }^{(23)}$.

Os estudos primários incluídos na primeira categoria investigaram a relação entre LPP e composição corporal. Ressalta-se que a maioria dos estudos foram realizados com pacientes idosos. Observa-se, para essa faixa etária, que dois estudos apresentaram uma relação de proteção contra LPP e a quantidade de massa gorda $^{(15-16)}$. Além disso, a redução da quantidade de água corporal esteve relacionada ao maior risco de $\operatorname{LPP}^{(13-14,16)}$.

Estudo realizado com idosos com e sem LPP evidenciou que não havia divergência na composição corporal dos grupos. Todavia, houve alteração dos níveis séricos de proteína ${ }^{(12)}$, o que, a longo prazo, poderia interferir na perda de massa celular e, consequentemente, da massa muscular dos pacientes idosos com LPP. Com a progressão da internação, estudo evidenciou perda de $20 \%$ de massa celular em pacientes idosos com LPP.

O IMC foi considerado como parâmetro de avaliação do risco para LPP no estudo quase experimental, em que foi elaborado um modelo matemático para determinação do risco de $\mathrm{LPP}^{(19)}$. 
Pesquisa que avaliou as melhores práticas para a prevenção de LPP em pacientes cirúrgicos considera que um critério determinante de risco para LPP é o IMC $<19$ ou $>40 \mathrm{~kg} / \mathrm{m}^{2}$. O IMC é importante para a escolha do posicionamento perioperatório e escolha da superfície de suporte a ser utilizada. A maioria das superfícies de suporte proporciona uma redistribuição da pressão em pacientes magros, mas podem não ser eficientes para pacientes obesos ${ }^{(24)}$.

Nos outros estudos apresentados na presente revisão, apesar de encontrarem relação da composição corporal com a ocorrência ou o risco de LPP, o IMC não foi um fator a ser considerado.

Estudo que objetivou revelar fatores de risco consistentes que podem contribuir para o desenvolvimento de LPP em pacientes submetidos a procedimentos cirúrgicos foi desenvolvido com dois grupos, dos quais um apresentou LPP na primeira semana após o procedimento e o outro não. O IMC médio foi de $29,6 \mathrm{~kg} / \mathrm{m}^{2}$ no grupo com LPP, enquanto no grupo sem LPP foi de $31,8 \mathrm{~kg} / \mathrm{m}^{2(7)}$.

Estudo longitudinal, realizado no norte da Itália, que avaliou a incidência de LPP e os fatores de risco associados em pacientes cirúrgicos, não encontrou associação entre a presença de LPP e valores de IMC ${ }^{(25)}$.

Estudo de coorte prospectivo, que identificou a prevalência e os fatores de risco associados ao desenvolvimento de LPP em pacientes submetidos a procedimentos cirúrgicos com duração superior a três horas, evidenciou maior incidência de LPP em homens em relação às mulheres. Os autores consideraram que esse achado pode estar relacionado à presença e distribuição de tecido adiposo, que o sexo masculino possui em menor quantidade em região sacral ${ }^{(26)}$. Essa característica foi observada no estudo 3, no qual os homens apresentaram maior quantidade de massa magra e menor quantidade de massa gorda ${ }^{(14)}$.

Em relação à categoria 2, foi observado que o estudo $6^{(17)}$ não observou uma relação da pressão de interface. Entretanto, o estudo $8^{(19)}$ evidenciou um comportamento divergente entre o risco de LPP para um grupo de indivíduos com mesmo IMC, o que pode sugerir que a composição de massa magra, água e gordura interfere nessa relação.

Em relação ao estudo experimental $9^{(20)}$, foram observados comportamentos diferentes das camadas de tecido gorduroso e muscular frente à exposição de tensões mecânicas. Esse comportamento modificou-se à medida que a espessura da camada muscular foi alterada. Pode-se pressupor com isso que a quantidade de massa magra e massa gorda possa ter influência na ação das tensões e, consequentemente, na etiologia da LPP.

Vale ressaltar a importância do conhecimento, pelos enfermeiros, dos fatores de risco relacionados à etiologia das LPP, independentemente do nível de complexidade dos pacientes, visto que essas possuem alta prevalência em hospitais, mas acometem também pacientes em domicílio. Estudo recente identificou a prevalência e caracterizou feridas crônicas junto a idosos assistidos na atenção básica, obtendo uma prevalência de $8 \%$, dos quais 5\% eram LPP, com tempo médio de existência de três anos ${ }^{(27)}$. Tais resultados merecem destaque, pois a LPP é uma realidade, mesmo em domicílio, o que exige que o enfermeiro seja capaz de implementar ações preventivas e de prestar assistência segura e livre de eventos adversos.

Como limitações da pesquisa, considera-se a delimitação do idioma nas línguas portuguesa, inglesa e espanhola, o que pode levar à exclusão de artigos que responderiam à pergunta norteadora, a escassez de estudos que relacionassem a composição corporal ou a pressão de interface com a ocorrência de lesão por pressão em indivíduos adultos, e ainda a heterogeneidade dos delineamentos dos estudos primários incluídos, fato que não possibilitou comparações entre os resultados.

Esta revisão contribui com evidências relevantes para a construção do conhecimento sobre a prática clínica dos enfermeiros, pois destaca fatores da composição corporal, além do índice de massa corporal, que podem influenciar no risco da ocorrência de lesão por pressão. A identificação de pacientes com maior risco de 
desenvolver lesão por pressão favorece a elaboração de planos de cuidados pautados na incorporação de evidências científicas na prática clínica e no processo de tomada de decisão, para prestar um cuidado seguro e de qualidade.

\section{Conclusão}

Entre as evidências encontradas, observa-se que a maioria dos estudos possuía delineamento descritivo analítico. Nesses estudos, o foco da avaliação foi os aspectos nutricionais de idosos, abrangendo composição corporal e avaliações séricas. Essas avaliações foram realizadas em idosos com e sem lesão.

Nesses estudos, a quantidade de água corporal foi relacionada à ocorrência de LPP. Maior quantidade de gordura corporal foi considerada como fator protetor para ocorrência de LPP em pacientes idosos.

Três pesquisas avaliaram a pressão de interface em relação à ocorrência ou risco de LPP. Essas pesquisas tiveram delineamentos quase experimentais ou experimentais laboratoriais. Constatou-se que a quantidade de massa muscular e água interfere na formação de LPP. Além disso, em grupos de indivíduos com idades variadas, uma mesma classificação nutricional obteve pressões de interface heterogêneas. Camadas musculares apresentam maior deformação quando submetidas à pressão de interface próximo à interface músculo-osso.

Pôde-se concluir que houve relação da composição corporal com a ocorrência de lesão por pressão, apresentando divergências entre adultos e idosos.

É importante destacar a necessidade de fortalecer a enfermagem baseada em evidência com vistas à qualidade da assistência e segurança do paciente. Sugere-se a realização de estudos futuros sobre a temática da presente revisão com rigor metodológico para prevenção de lesão por pressão e melhoria da assistência em saúde.

\section{Colaborações:}

1 - concepção, projeto, análise e interpretação dos dados: Jéssica Gomes da Silva, Karoline Faria de Oliveira, Maria Beatriz Guimarães Ferreira, Flávia Ana Pacheco, Isadora Braga Calegari e Maria Helena Barbosa;

2 - redação do artigo e revisão crítica relevante do conteúdo intelectual: Jéssica Gomes da Silva, Karoline Faria de Oliveira, Maria Beatriz Guimarães Ferreira, Flávia Ana Pacheco, Isadora Braga Calegari e Maria Helena Barbosa;

3 - aprovação final da versão a ser publicada: Jéssica Gomes da Silva, Karoline Faria de Oliveira, Maria Beatriz Guimarães Ferreira, Flávia Ana Pacheco, Isadora Braga Calegari e Maria Helena Barbosa.

\section{Referências}

1. McInnes E, Jammali-Blasi A, Bell-Syer SEM, Dumville JC, Middleton V, Cullum N. Support surfaces for pressure ulcer prevention. Cochrane Database Syst Rev. 2015;3(9):CD001735. doi: 10.1002/14651858.CD001735.pub5

2. Scott SM. Creating a strategic plan for perioperative pressure ulcer prevention. AORN J. 2016 Apr;103(4):13-14.

3. Kwong EW, Hung MS, Woo K. Improvement of pressure ulcer prevention care in private forprofit residential care homes: an action research study. BMC Geriatr [Internet]. 2016 [cited 2017 Apr 17];16(1):192. Available from: https://www.ncbi. nlm.nih.gov/pubmed/27884131

4. Nguyen KH, Chaboyer W, Whitty JA. Pressure injury in Australian public hospitals a cost-ofillness study. Aust Health Rev [Internet]. 2015 May [cited 2017 May 14];39(3):329-36. Available from: https://www.ncbi.nlm.nih.gov/pubmed/25725696

5. Coleman S1, Nixon J, Keen J, Wilson L, McGinnis E, Dealey C, et al. A new pressure ulcer conceptual framework. J Adv Nurs [Internet]. 2014 Oct [cited 2017 Apr 12];70(10):2222-34. Available from: https://www.ncbi.nlm.nih.gov/pubmed/24684197 
6. Association of periOperative Registered Nurses. Guideline for positioning the patient. In: Guidelines for Perioperative Practice. Colorado, EUA; 2017.

7. Engels D, Austin M, McNichol L, Fencl J. Pressure ulcers: factors contributing to their development in the OR. AORN J [Internet]. 2016 Mar [cited 2017 Apr 17];103(3):271-81. Available from: https:// www.ncbi.nlm.nih.gov/pubmed/26924365

8. Mendes KDS, Silveira RCCP, Galvão CM. Integrative literature review: a research method to incorporate evidence in health care and nursing. Texto Contexto Enferm [Internet]. 2008 [cited 2017 May 12];17(4):758-64. Available from: http://www.scielo.br/scielo.php?script=sci_ arttext\&pid=S0104-07072008000400018

9. Melnyk BM, Fineout-Overholt E. Making the case for evidence-based practice. In: Melnyk BM, Fineout-Overholt E, editors. Evidence-based practice in nursing and healthcare: a guide to best practice. Philadelphia: Lippincott Williams and Wilkins; 2005. p. 3-24.

10. Ursi ES, Galvão CM. Perioperative prevention of skin injury: an integrative literature review. Rev Lat Am Enfermagem [Internet]. 2006 [cited 2017 Jun 10];14(1):124-31. Available from: http://www.scielo.br/scielo.php?script=sci_ abstract\&pid=S0104-11692006000100017\&lng=en $\& n r m=i s o \& t l n g=e n$

11. Politi DF, Beck CT. Fundamentos de pesquisa em enfermagem: avaliação de evidências para a prática de enfermagem. 7a ed. Porto Alegre: Artmed; 2016.

12. Sergi G, Coin A, Mulone S, Castegnaro E, Giantin V, Manzato E, et al. Resting energy expenditure and body composition in bedridden institutionalized elderly women with advanced-stage pressure sores. J Gerontol A Biol Sci Med Sci [Internet]. 2007 [cited 2017 Apr 17];62(3):317-22. Available from: https://www.ncbi.nlm.nih.gov/pubmed/17389730

13. Lobo A. Factores de riesgo en el desarrollo de úlceras de presión y sus implicaciones en la calidad de vida. Rev bras geriatr gerontol [Internet]. 2008 [cited 2017 Jun 10];11(3):405-18. Available from: http://www.scielo.br/scielo.php?pid=\$180998232008000300405\&script=sci_abstract\&tlng

14. Fiedler M, Gerhardt LC, Derler S, Bischofberger G, Hürny C, Münzer T. Assessment of biophysical skin properties at different body sites in hospitalized old patients: results of a pilot study. Gerontology
[Internet]. 2012 [cited 2017 Apr 17];58(6):513-7. Available from: https://www.ncbi.nlm.nih.gov/ pubmed/22487874

15. Bouillanne O, Dupont-Belmont C, Hay P, HamonVilcot B, Cynober L, Aussel C. Fat mass protects hospitalized elderly persons against morbidity and mortality. Am J Clin Nutr [Internet]. 2009 [cited 2017 May 12];90(3):505-10. Available from: https:// www.ncbi.nlm.nih.gov/pubmed/19640947

16. Hengstermann S, Fischer A, Steinhagen-Thiessen E, Schulz RJ. Nutrition status and pressure ulcer: what we need for nutrition screening. JPEN J Parenter Enteral Nutr [Internet]. 2007 [cited 2017 Apr 17];31(4):288-94. Available from: https://www. ncbi.nlm.nih.gov/pubmed/17595437

17. Cho KH, Beom J, Yuk JH, Ahn SC. The Effects of Body Mass Composition and Cushion Type on Seat-Interface Pressure in Spinal Cord Injured Patients. Ann Rehabil Med [Internet]. 2015 [cited 2017 Apr 17];39(6):971-9. Available from: https:// www.ncbi.nlm.nih.gov/pubmed/26798612

18. Wagner DR, Jeter KF, Tintle T, Martin MS, Long JM. Bioelectrical impedance as a discriminator of pressure ulcer risk. Adv in Wound Care [Internet]. 1996 [cited 2017 Jun 10];9(2):30-7. Available from: https://journals.lww.com/aswcjournal/ Abstract/1996/09020/Bioelectrical_Impedance_ as_a_Discriminator_of.7.aspx

19. Mishu MC, Schroeder JW. Modelling of pressure ulcer (PU) risk prediction system. Science and Information Conference. 2015 July:650-6. doi: 10.1109/SAI.2015.7237211

20. Luboz V, Petrizelli M, Bucki M, Diot B, Vuillerme N, Payan Y. Biomechanical modeling to prevent ischial pressure ulcers. J Biomech [Internet]. 2014 Jul [cited 2017 May 12];47(10):2231-6. Available from: https://www.ncbi.nlm.nih.gov/pubmed/24873863

21. National Pressure Ulcer Advisory Panel. European Pressure Ulcer Advisory Panel. Pan Pacific Pressure Injury Alliance. Prevention and Treatment of Pressure Ulcers: Quick Reference Guide. Haesler E, editor. Cambridge Media: Osborne Park, Western Australia; 2016.

22. Wong A, Chew A, Wang CM, Ong L, Zhang SH, Young S. The use of a specialised amino acid mixture for pressure ulcers: a placebo-controlled trial. J Wound Care [Internet]. 2014 May [cited 2017 Apr 17];23(5):259-60, 262-4, 266-9. Available from: https://www.ncbi.nlm.nih.gov/pubmed/24810310 
23. Cereda E, Klersy C, Serioli M, Crespi A, D'Andrea F, OligoElement Sore Trial Study Group. A nutritional formula enriched with arginine, zinc, and antioxidants for the healing of pressure ulcers: a randomized trial. Ann Intern Med [Internet]. 2015 Feb [cited 2017 Jun 10];162(3):16774. Available from: https://www.ncbi.nlm.nih.gov/ pubmed/25643304

24. Cherry C, Moss J. Best practices for preventing hospital-acquired pressure ulcer injuries in surgical patients. Can Oper Room Nurs J [Internet]. 2011 Mar [cited 2017 May 12];29(1):6-26. Available from: https://www.ncbi.nlm.nih.gov/pubmed/21434513

25. Bulfone G, Marzoli I, Quattrin R, Fabbro C, Palese A. A longitudinal study of the incidence of pressure sores and the associated risks and strategies adopted in Italian operating theatres. J Perioper Pract [Internet]. 2012 Feb [cited 2017
Apr 17];22(2):50-6. Available from: https://www. ncbi.nlm.nih.gov/pubmed/22724304

26. Primiano M, Friend M, McClure C, Nardi S, Fix L, Schafer $\mathrm{M}$, et al. Pressure ulcer prevalence and risk factors during prolonged surgical procedures. AORN J [Internet]. 2011 Dec [cited 2017 Apr 17];94(6):555-66. Available from: https://www. ncbi.nlm.nih.gov/pubmed/22118201

27. Vieira CPB, Furtado AS, Almeida PCD, Luz MHBA, Pereira AFM. Prevalência e caracterização de feridas crônicas em idosos assistidos na atenção básica. Rev baiana enferm [Internet]. 2017 [cited 2019 Jul 18];31(3):e17397. Available from: https:// portalseer.ufba.br/index.php/enfermagem/article/ view/17397

Recebido: 2 de dezembro de 2018

Aprovado: 20 de agosto de 2019

Publicado: 23 de janeiro de 2020

A Revista Baiana de Enfermagem utiliza a Licença Creative Commons - Atribuição-NãoComercial 4.0 Internacional.

https://creativecommons.org/licenses/by-nc/4.0/

Este artigo é de acesso aberto distribuído sob os termos da Licença Creative Commons (CC BY-NC).

Esta licença permite que outros remixem, adaptem e criem a partir do seu trabalho para fins não comerciais. Embora os novos trabalhos tenham de lhe atribuir o devido crédito e não possam ser usados para fins comerciais, os usuários não têm de licenciar esses trabalhos derivados sob os mesmos termos. 